\title{
Anhang: Sterilisationsstatistik
}

Unter den Akten des Reichsjustizministeriums befindet sich die folgende, bisher unveröffentlichte Sterilisationsstatistik zu den Jahren 1934-1936 ${ }^{1}$. Sie benennt für sämtliche Oberlandesgerichts- bzw. Erbgesundheitsobergerichtsbezirke in Deutschland die Zahl der jeweils anhängigen Sterilisationsverfahren (Antr) und demgegenüber die Zahlen der Anordnungen bzw. Ablehnungen (Steri/nSteri).

\begin{tabular}{|c|c|c|c|c|c|c|c|c|c|}
\hline \multirow[b]{2}{*}{ Bezirk } & \multicolumn{3}{|c|}{1934} & \multicolumn{3}{|c|}{1935} & \multicolumn{3}{|c|}{1936} \\
\hline & Antr & Steri & nSteri & Antr & Steri & nSteri & Antr & Steri & nSteri \\
\hline Bamberg & 1346 & 811 & 115 & 1387 & 1024 & 191 & 1817 & 1216 & 242 \\
\hline Berlin & 6562 & 4482 & 247 & 7320 & 6297 & 863 & 6968 & 4909 & 1079 \\
\hline Braunschweig & 450 & 401 & 24 & 637 & 502 & 77 & 498 & 396 & 102 \\
\hline Breslau & 5015 & 3594 & 224 & 6080 & 5043 & 609 & 5652 & 4316 & 875 \\
\hline Celle & 4554 & 3838 & 209 & 5118 & 4245 & 487 & 4764 & 3783 & 648 \\
\hline Darmstadt & 2009 & 1387 & 65 & 1641 & 1313 & 113 & 1817 & 1608 & 188 \\
\hline Dresden & 8222 & 6013 & 333 & 7511 & 6456 & 572 & 6796 & 5472 & 884 \\
\hline Düsseldorf & 4143 & 2854 & 170 & 3725 & 3212 & 333 & 4115 & 2979 & 434 \\
\hline Frankfurt (M.) & 1295 & 826 & 117 & 1612 & 1284 & 217 & 1826 & 1362 & 342 \\
\hline Hamburg & 3934 & 3401 & 163 & 4763 & 3373 & 297 & 3954 & 3184 & 601 \\
\hline Hamm (Westf.) & 5875 & 4137 & 393 & 7394 & 6006 & 1004 & 6763 & 5415 & 1049 \\
\hline Jena & 3002 & 2326 & 247 & 3366 & 2724 & 273 & 3641 & 2461 & 432 \\
\hline Karlsruhe & 6161 & 5060 & 357 & 4497 & 3509 & 364 & 3554 & 2764 & 365 \\
\hline Kassel & 1617 & 1144 & 62 & 1815 & 1613 & 155 & 1503 & 1109 & 2455 \\
\hline Kiel & 2061 & 1768 & 115 & 2077 & 1440 & 170 & 1878 & 1557 & 219 \\
\hline Köln & 4411 & 2555 & 245 & 5369 & 4158 & 597 & 5925 & 4390 & 875 \\
\hline Königsberg & 3296 & 2429 & 100 & 3141 & 2866 & 277 & 2842 & 2171 & 308 \\
\hline Marienwerder & 608 & 437 & 36 & 877 & 724 & 99 & 981 & 607 & 110 \\
\hline München & 3142 & 2169 & 188 & 3495 & 2718 & 77 & 4018 & 2893 & 433 \\
\hline Naumburg (S.) & 4610 & 3372 & 194 & 4420 & 3850 & 345 & 4668 & 3756 & 449 \\
\hline Nürnberg & 1815 & 1239 & 209 & 1509 & 1097 & 224 & 1603 & 967 & 171 \\
\hline Oldenburg & 907 & 731 & 161 & 709 & 456 & 140 & 448 & 257 & 52 \\
\hline Rostock & 1490 & 1344 & 72 & 1766 & 1394 & 114 & 1311 & 947 & 180 \\
\hline Stettin & 2957 & 2200 & 248 & 2759 & 2494 & 314 & 2636 & 1817 & 347 \\
\hline Stuttgart & 3447 & 2611 & 449 & 3333 & 2244 & 578 & 4193 & 2776 & 696 \\
\hline Zweibrücken & 1675 & 1335 & 131 & 1872 & 1718 & 186 & 2092 & 1594 & 293 \\
\hline insgesamt & 84604 & 62463 & 4874 & 88139 & 71760 & 8976 & 86254 & 64646 & 11619 \\
\hline
\end{tabular}

\footnotetext{
${ }^{1}$ BAK, R 22 RJuM 1933, S. 355 f.
} 
Zur besseren Vergleichbarkeit haben wir diese absoluten Zahlen umgerechnet auf die Zahl der anhängigen Verfahren pro Kopf der Bevölkerung ${ }^{2}$ in Promille (Verfahrenshäufigkeit, Antr), die Sterilisationsbeschlüsse (Steri) pro Kopf der Bevölkerung in Promille und das Verhältnis der Sterilisationsablehnungen zu den abgeschlossenen Verfahren in Prozent (Ablehnungsquote Aqu) ${ }^{3}$.

\begin{tabular}{|c|c|c|c|c|c|c|c|c|c|}
\hline & & 1934 & & & 1935 & & & 1936 & \\
\hline & Antr & Aqu & Steri & Antr & Aqu & Steri & Antr & Aqu & Steri \\
\hline Bamberg & 0,85 & 11,4 & 0,51 & 0,88 & 8,31 & 0,65 & 1,15 & 16,6 & 0,77 \\
\hline Berlin & 0,94 & 5,3 & 0,64 & 1,05 & 12,1 & 0,90 & 1,00 & 18,0 & 0,85 \\
\hline Braunschweig & 0,88 & 5,65 & 0,78 & 1,24 & 13,6 & 0,98 & 0,97 & 20,4 & 0,96 \\
\hline Breslau & 1,06 & 5,68 & 0,76 & 1,29 & 10,8 & 1,07 & 1,20 & 16,9 & 0,92 \\
\hline Celle & 1,27 & 5,16 & 1,07 & 1,43 & 10,3 & 1,18 & 1,33 & 14,6 & 1,05 \\
\hline Darmstadt & 1,41 & 4,48 & 0,97 & 1,15 & 7,92 & 0,92 & 1,27 & 10,4 & 1,13 \\
\hline Dresden & 1,58 & 5,24 & 1,16 & 1,47 & 8,14 & 1,24 & 1,31 & 12,7 & 1,05 \\
\hline Düsseldorf & 1,20 & 5,62 & 0,82 & 1,08 & 8,21 & 0,93 & 1,19 & 13,4 & 0,86 \\
\hline Frankfurt (M.) & 0,97 & 14,2 & 0,62 & 1,21 & 14,5 & 0,96 & 1,37 & 20,0 & 1,02 \\
\hline Hamburg & 2,22 & 4,57 & 1,92 & 2,68 & 8,09 & 1,90 & 2,23 & 15,8 & 1,79 \\
\hline Hamm (Westf.) & 1,00 & 8,68 & 0,70 & 1,26 & 14,3 & 1,03 & 1,15 & 16,2 & 0,92 \\
\hline Jena & 1,76 & 2,60 & 1,36 & 1,97 & 9,11 & 1,59 & 2,05 & 14,9 & 1,44 \\
\hline Karlsruhe & 2,55 & 6,59 & 2,10 & 1,86 & 9,40 & 1,45 & 1,47 & 11,6 & 1,14 \\
\hline Kassel & 1,42 & 5,14 & 0,98 & 1,60 & 8,77 & 1,41 & 1,32 & 18,0 & 0,98 \\
\hline Kiel & 1,30 & 6,11 & 1,11 & 1,83 & 10,0 & 1,27 & 1,65 & 12,3 & 1,37 \\
\hline Köln & 1,03 & 8,75 & 0,60 & 1,25 & 12,5 & 0,97 & 1,38 & 16,6 & 2,02 \\
\hline Königsberg & 1,60 & 3,95 & 1,18 & 1,53 & 8,81 & 1,39 & 1,38 & 12,4 & 1,06 \\
\hline Marienwerder & 1,02 & 8,28 & 0,74 & 1,47 & 12,0 & 1,22 & 1,65 & 15,3 & 1,02 \\
\hline München & 0,93 & 7,98 & 0,64 & 1,04 & 12,2 & 0,81 & 1,19 & 13,0 & 0,86 \\
\hline Naumburg (S.) & 1,23 & 5,44 & 9,90 & 1,17 & 8,22 & 1,02 & 1,24 & 10,7 & 1,00 \\
\hline Nürnberg & 1,04 & 14,4 & 0,77 & 0,86 & 17,0 & 0,63 & 0,92 & 15,0 & 0,55 \\
\hline Oldenburg & 1,94 & 11,4 & 1,56 & 1,51 & 23,5 & 0,98 & 0,96 & 16,8 & 0,55 \\
\hline Rostock & 1,85 & 5,08 & 1,70 & 2,19 & 7,56 & 1,73 & 1,63 & 16,0 & 1,18 \\
\hline Stettin & 1,54 & 9,30 & 1,15 & 1,44 & 11,2 & 1,17 & 1,37 & 16,0 & 0,95 \\
\hline Stuttgart & 1,24 & 14,7 & 0,94 & 1,20 & 20,4 & 0,81 & 1,40 & 20,0 & 1,00 \\
\hline Zweibrücken & 1,70 & 8,94 & 1,36 & 1,91 & 9,77 & 1,74 & 2,12 & 15,5 & 1,26 \\
\hline insgesamt & 1,28 & 7,24 & 0,94 & 1,33 & 11,1 & 1,08 & 1,30 & 15,2 & 0,98 \\
\hline
\end{tabular}

Teilweise identisch mit der (ersten) Statistik ist das Datenmaterial, das Karl Bonhoeffer in seiner Untersuchung zur Sterilisationspraxis in Deutschland 1949 vorgelegt hat ${ }^{4}$. Er hatte offensichtlich die Daten der Statistik über die Jahre 1934 35 vor Augen 5 .

\footnotetext{
2 Eine nach Oberlandesgerichtsbezirken geordnete Verteilung der Einwohnerzahlen (Stand 1935) findet sich im Statistischen Jahrbuch 1936, S. 554.

${ }^{3}$ Hierzu wurden die Sterilisationsablehnungen durch die Summe aus Sterilisationsbeschlüssen und Sterilisationsablehnungen geteilt, d.h. die bei Jahresende nicht abgeschlossenen Verfahren nicht einbezogen.

${ }^{4}$ Vgl. Bonhoeffer, Rückblick Sterilisationsgesetz; siehe S.112.

${ }^{5}$ Als Vermittler fungierte vermutlich Dohnanyi, siehe S. 103.
} 
Anhand der hier vorgelegten Gesamtstatistik erhärtet sich seine These, daß die Sterilisationspraxis regional sehr unterschiedlich ausgeprägt war. Die Quoten über die Verfahrenshäufigkeit schwanken 1935 zwischen 0,88 Promille im Bezirk des EOG Bamberg und 2,68 Promille im Bezirk des EOG Hamburg. Bonhoeffer beobachtete, daß "in Landschaften, in denen besonders eifrige Anhänger der sog. Erbpflege im Gesundheitswesen einen maßgeblichen Einfluß hatten, die Zahl der Sterilisationen erheblich über die Zahlen anderer Bezirke mit ähnlicher Bevölkerungszahl hinausging“6.

Die regionalen Unterschiede können nicht durch den Unterschied zwischen Stadt und Land erklärt werden, denn der städtisch geprägte Bezirk des EOG Hamburg (umfassend Hamburg und Bremen) hat relativ hohe, die ebenfalls städtisch geprägten Bezirke des EOG München (umfassend München und Umland) und EOG Berlin (umfassend Berlin-Brandenburg) hatten relativ niedrige Zahlen. Auch konfessionelle Unterschiede haben keine wesentliche Rolle gespielt, denn überwiegend protestantische Regionen wie Hamburg-Bremen und Thüringen (EOG Jena) führen die Statistik ebenso an wie das überwiegend katholisch geprägte Baden (EOG Karlsruhe).

${ }^{6}$ Vgl. Bonhoeffer, Rückblick Sterilisationsgesetz, S.4. Wie von seiten der "Erbpflege“ mitunter mit der Frage umgegangen wurde, illustriert das Diensttagebuch Gürtners: Demnach forderte das "Landesamt für Rassepflege" in Thüringen, daß 30000 Personen sterilisiert werden müßten. Wie der Jenaer Oberlandesgerichtspräsident dem Reichsjustizministerium meldete, sei man dabei folgendermaßen vorgegangen: „Es wurde[n] [...] in bewußter Abweichung von anderen Ländern für die LG-Bezirke je mehrere Erbgesundheitsgerichte errichtet." Diese Meldung kommentierte Dohnanyi: „Thüringen, Einwohnerzahl 1659510 (also jeder 55. war zu sterilisieren) “; vgl. National Archives, M 978, Diensttagebuch Gürtner, Eintrag vom 26.11. 1936. 
